\title{
Analysis of Wireless Power Transfer Using Metamaterial Slabs Made of Ring Resonators at $13.56 \mathrm{MHz}$
}

\author{
TaekKyu Oh $\cdot$ Bomson Lee*
}

\begin{abstract}
This paper analyzes the effects of metamaterial slabs with negative permeability when applied to a two-loop wireless power transmission (WPT) system, both in theory and electromagnetic (EM) simulations. The analysis of magnetic flux focusing provided here assumes quasi-magnetostatics or magnetostatics. The slab structures with negative permeability have been realized using the periodically arrayed ring resonators (RRs) at $13.36 \mathrm{MHz}$. Some examples with ideal lossless slabs of $-1,-2$, and -3 showed a great enhancement of WPT efficiencies when compared with the free space cases. However, practical lossy slabs made of planar copper RRs did not show significant enhancement of WPT efficiencies due to the relatively high losses in the ring resonator (or in the slab consisting of RRs) near the resonant frequency.
\end{abstract}

Key Words: Magnetic Flux Focusing, Metamaterial, Negative Permeability, Ring Resonator, Split Ring Resonator.

\section{INTRODUCTION}

In recent years, wireless power transmission (WPT) technology has become one of the fastest and highest impact technologies. The concept of WPT was initiated by N. Tesla in 1914 , but it had not resulted in any practical applications until recently, due to its low efficiency. A magnetically coupled WPT based on the coupled mode theory was first investigated in 2007 by Prof. Soljacic and his research group at MIT [1]. However, a given WPT system typically consisting of two resonators with a certain separation in free space has a limit of WPT efficiencies. Much research has been performed in an attempt to overcome this problem. For example, metamaterial has been considered as one solution that can address the low efficiency problem.

The realization of media that have negative permittivity and permeability (also called left-handed metamaterial) has become feasible since Pendry et al. [2] proposed a new me- thod in 1999 that used an array of thin wires and split ring resonators (SRRs). A focusing problem for a magnetostatic case with an effective permeability of -1 was treated in [3]. The present paper derives a simple design formula for the case with general negative permeability based on $[4,5]$. The provided formula facilitates the design of two-loop WPT configurations that employ metamaterial slabs with arbitrary negative effective permeability (for instance, $-1,-2$, and -3 ). The WPT with lossless and lossy slabs are examined in terms of their $S$-parameters and the efficiencies based on them.

The metamaterial slabs are assumed to be ideally isotropic or realized by periodically arrayed ring resonators (RRs). The effects of losses when using RRs are also estimated.

\section{Wpt System Using Metamaterial Slabs For} Magnetic Flux Focusing

Manuscript received October 18, 2013 ; Revised November 18, 2013 ; Accepted November 26, 2013. (ID No. 20131018-040J)

Department of Electronics and Radio Engineering, Kyung Hee University, Yongin, Korea.

"Corresponding Author: Bomson Lee (e-mail: bomson@khu.ac.kr)

This is an Open-Access article distributed under the terms of the Creative Commons Attribution Non-Commercial License (http://creativecommons.org/licenses/by-nc/3.0) which permits unrestricted non-commercial use, distribution, and reproduction in any medium, provided the original work is properly cited.

(C) Copyright The Korean Institute of Electromagnetic Engineering and Science. All Rights Reserved. 
At the very low frequencies (several $\mathrm{MHz}$ down to $\mathrm{kHz}$ ) used in WPT systems, the system size is very small compared with the wavelength. For this case, the quasistatics may be the governing rule. The loop resonator (loop antenna with a chip capacitor loaded onto it) used in this work may be best modeled in the framework of quasi-magnetostatics. It may also be understood as a magnetic dipole. Fig. 1 shows the magnetically coupled WPT system using the metamaterial slabs with negative permeability. A generalized formula based on the magnetostatic field refraction [4] has been derived and is given by

$$
\frac{\tan \theta_{2}}{\tan \theta_{1}}=\frac{a}{a^{\prime}}=\frac{b}{b^{\prime}}=-\mu_{r}
$$

where $\theta_{1}$ is the incident angle and $\theta_{2}$ is the refraction angle, $a, a^{\prime}, b, b^{\prime}$ and are distances as defined in Fig. $1, d$ is the distance between the two loops $\left(d=a+a^{\prime}+b+b^{\prime}\right)$, $b$ is the height of the slab determined to capture as much magnetic fields from the loop as possible, and $\mu_{r}$ is the relative effective permeability usually realized with RRs or SRRs. The sum of $a^{\prime}$ and $b^{\prime}$ is the width $(s)$ of the slab. The focusing of the fields from the loop mainly depends on the permeability of the slab, and not on the permittivity since magnetic fields are dominant in the near region of the loop.

Negative permeability can also be determined by using Eq. (1) for a given thickness of the slab $(s)$ and a distance between two loops $(d)$. Note also that the same focusing effect takes place as long as it is located between the two loops and the slab height is sufficiently long, independent of its position.

\section{Simulation Results And Discussions}

The WPT system used in electromagnetic (EM) simulations is shown in Fig. 1. The two loop-resonators are made of copper and are designed at $13.56 \mathrm{MHz}$. The radius $\left(r_{1}\right)$ of the loop is $10 \mathrm{~cm}$ and the radius $\left(r_{\text {ring }}\right)$ of the ring is 1 $\mathrm{cm}$. The inductance of the loop is $47.63 \mathrm{nH}$, the capacitance of the loaded chip capacitor is $383 \mathrm{pF}$, the ohmic resistance of the loop is $0.025 \Omega$, and the Q-factor is 1,838 at the resonant frequency of $13.56 \mathrm{MHz}$. Table 1 summarizes the EM-simulated WPT efficiencies for different $a, s$, and load

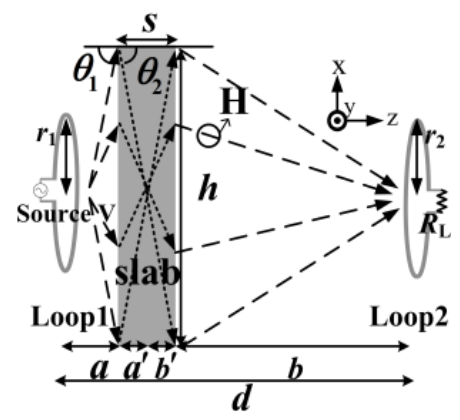

Fig. 1. Magnetically coupled wireless power transmission with metamaterial slab of negative permeability. resistance $\left(R_{L}\right)$ with a fixed $d=48 \mathrm{~cm}$. The optimum load $\left(R_{L, o p t}\right)$ for a maximum efficiency when the two loops are in free space is given by

$$
R_{L, o p t}=R_{2} \sqrt{1+k^{2} Q_{1} Q_{2}}
$$

where $R_{2}$ is the resistance of the second loop, $k$ is the coupling coefficient, $Q_{1}$ and $Q_{2}$ are the quality factors of the first and second resonator, respectively. The expression (2) is derived using circuit theory and has been checked to be equivalent to the expression in [2] written in terms of coupled mode theory. The optimum load $\left(R_{L, \text { opt }}\right)$ for a maximum efficiency when using the slab has been determined from EM simulations. The WPT efficiencies have been evaluated with $\left|S_{21}\right|^{2} /\left(1-\left|S_{11}\right|^{2}\right)$, which remains the same for any reference impedance of port 1 .

Table 1 shows that the WPT efficiencies are greatly enhanced compared to the free space case due to the effect of magnetic field focusing when the lossless slabs are inserted between the two loops.

In addition, the results show that the use of the optimum load (2) is crucial for further enhancement of the WPT efficiencies. Fig. 2 shows the EM-simulated $S$-parameters for the cases in Table 1. Fig. 2(a) shows the $S$-parameters without the slab and with a slab having $\mu_{r}=-2$ with $a=$ $16 \mathrm{~cm}$. For the case with the slab, the resonant frequency has shifted somewhat downward. The efficiencies in Table 1 have been evaluated at about $12.3 \mathrm{MHz}$. This can be tuned back to the required resonant frequency of $13.56 \mathrm{MHz}$ by adjusting the capacitance of the chip capacitor placed on the loop with almost the same efficiency. Fig. 2(b) shows the EM-simulated $S$-parameters for different distances $(a$ and $b$ ) and different values of permeability. For the cases where $\mu$ $=-2$ and $-3, s$ is determined to be 16 and $12 \mathrm{~cm}$, respectively, using (1). This result also indicates that the $S$-parameters do not depend on the position $a$ of the lossless slab.

Table 1. Comparison of WPT efficiencies when using lossless slabs $\left(f_{0}=13.56 \mathrm{MHz}, r_{1}=10 \mathrm{~cm}, d=48 \mathrm{~cm}, Q=1,838\right)$

\begin{tabular}{cccccc}
\hline \multirow{2}{*}{$\mu_{r}$} & $s(\mathrm{~cm})$ & $a(\mathrm{~cm})$ & $b(\mathrm{~cm})$ & \multicolumn{2}{c}{ Efficiency (\%) } \\
\cline { 5 - 6 } $\begin{array}{c}\text { (free } \\
\text { space) }\end{array}$ & - & - & - & 0.02 & $73(0.2)$ \\
& & & & & \\
-1 & 24 & 12 & 60 & 97 & $99(9.5)$ \\
& 24 & 8 & 60 & 98 & $99(9.5)$ \\
-2 & 16 & 16 & 80 & 90 & $98(7.6)$ \\
& 16 & 8 & 80 & 94 & $98(7.6)$ \\
-3 & 12 & 18 & 100 & 70 & $97(1.5)$ \\
& 12 & 8 & 100 & 74 & $97(1.5)$ \\
\hline
\end{tabular}

WPT $=$ wireless power transmission, $\mu_{r}=$ permeability of the slab, $s=$ slab width, $a=$ distance, $h=$ slab height, $R_{L}=$ load resistance. 


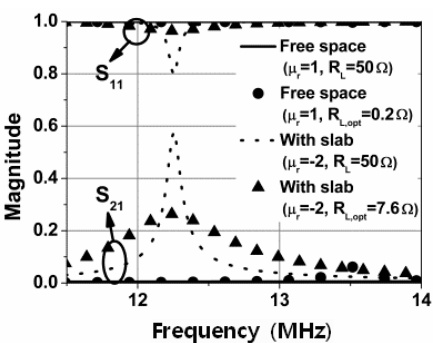

(a)

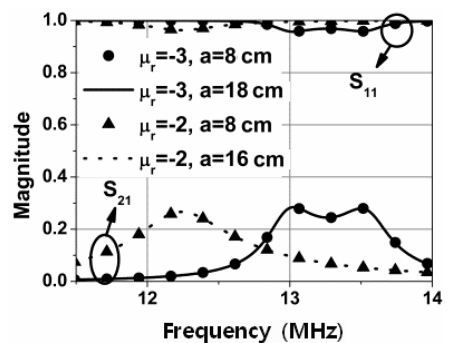

(b)
Fig. 2. Comparison of $\left|S_{11}\right|$ 's and $\left|S_{21}\right|$ 's as a function of frequency for the cases in Table 1. (a) Cases without and with slab $\left(\mu_{r}=-2\right)$ when $d=48 \mathrm{~cm}, s=16 \mathrm{~cm}$, and $a=16 \mathrm{~cm}$. (b) Cases with slabs $\left(\mu_{r}=-2,-3\right)$ for different $a$ 's.

Now, we deal with the case of $\mu_{r}=-1$ slabs considering loss effects. Fig. 3(a) shows the unit cell structure designed to have an isotropic effective permeability behavior. The copper ring resonator, used in the EM simulation has been designed to have the effective relative permeability of -1 at $13.56 \mathrm{MHz}$. Its inductance is $10.8 \mathrm{nH}$ and the capacitance of the inserted chip capacitor is $1.26 \mathrm{pF}$. The side length of the unit-cell is $12 \mathrm{~cm}$. The radii and thickness of the ring resonator are 4 and $1 \mathrm{~cm}$, respectively. Fig. 3(b) shows the extracted $\operatorname{Re}\left[\mu_{r}\right]$ and $\operatorname{Im}\left[\mu_{r}\right]$, which are -1 and 0.23 at the design frequency of $13.56 \mathrm{MHz}$, respectively. In Fig. 3(c), we compare the EM-simulated $S$-parameters for an ideal isotropic slab $\left(\mu_{r}=-1-j 0.23\right.$ irrespective of frequencies) with the EM-simulated and measured $S$-parameters for the RR bulk structure based on the configuration given by Fig. 1. For these simulations, $r_{1}=r_{2}=5 \mathrm{~cm}, d=24 \mathrm{~cm}, a=b=$ $6 \mathrm{~cm}, s=12 \mathrm{~cm}$, and the $Q$-factor of each loop is 473 . Although the $S$-parameters for the cases of highly dispersive RR bulk structures are far more dependent on frequencies than are those of the ideal isotropic slab where permeability is independent of frequencies, they are still in good agreement at $13.36 \mathrm{MHz}$. The slight difference between the EM-simulated and the measured $S$-parameters for RR bulk structures is judged to arise from fabrication errors. In Table 2 , we summarize the WPT efficiencies for the various cases including those in Fig. 3(c) when $R_{L}=50 \Omega$ and $R_{L}=$ $R_{L, o p t}$. For the case of the ideal lossless slab $\left(\mu_{r}=-1\right)$, a significant improvement in efficiencies is achieved up to $96 \%$, due to the effect of magnetic field focusing. For the lossy slabs with the imaginary part of 0.23 , however, the efficiencies decrease to about $20 \%$ due to absorption of power in the slabs. The critical imaginary part, where the maximum efficiency becomes that of free space (32\%), is 0.07 . The RRs used for this work is a planar type. If a ring type RR is employed, the loss effect decreases and a higher WPT efficiency may be obtained.

\section{Ii. CONCLUSION}

The effects of magnetic flux focusing when using a slab

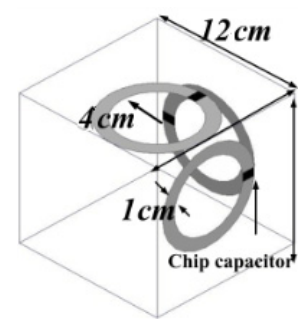

(a)

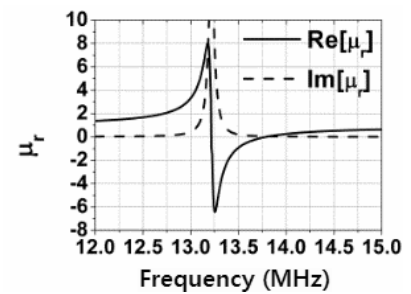

(b)

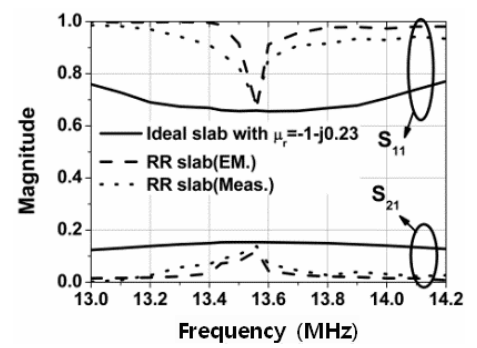

(c)

Fig. 3. Characteristics of $\mathrm{RR}$ and comparison of $S$-parameters $(d$ $=24 \mathrm{~cm}, a=b=6 \mathrm{~cm}, s=12 \mathrm{~cm}$ ). (a) Isotropic unit cell of RR. (b) Extracted $\mu_{r}$ for RR bulk. (c) Comparison of $S$-parameters for the cases of using ideal slab and RR bulks when $R_{L}=50 \Omega$.

Table 2. Summary of the WPT efficiencies $(d=24 \mathrm{~cm}, a=b=6$ $\mathrm{cm}, s=12 \mathrm{~cm}$ )

\begin{tabular}{ccc}
\hline \multirow{2}{*}{ Material } & \multicolumn{2}{c}{ Efficiency (\%) } \\
\cline { 2 - 3 } & $R_{L}=50 \Omega$ & $R_{L}=R_{L, o p t}$ \\
\hline Free space $\left(\mu_{r}=1\right)$ & 0 & $32(0.1)$ \\
Ideal lossless slab $\left(\mu_{r}=-1\right)$ & 80 & $97(17)$ \\
Ideal lossy slab $\left(\mu_{r}=-1-j 0.23\right)$ & 3.4 & $18.9(7)$ \\
$\quad$ Simulated RR bulk & 3 & $18.5(6.8)$ \\
$\left(\mu_{r}=-1-j 0.23\right.$ at $\left.13.56 \mathrm{MHz}\right)$ & & \\
$\quad$ Fabricated RR bulk & 3.9 & $22.2(6.8)$ \\
$\left(\mu_{r}=-1-j 0.23\right.$ at $\left.13.56 \mathrm{MHz}\right)$ & &
\end{tabular}

$\mathrm{WPT}=$ wireless power transmission, $\mathrm{RR}=$ ring resonators.

with negative permeability in WPT systems have been analyzed based on a formulation assuming magnetostatics. For the lossless slabs with $\mu_{r}=-1,-2$, and -3 , the WPT efficiencies are greatly improved to close to $100 \%$ due to the effect of focusing. For the lossy slabs consisting of planar type RRs, where effective permeability has been evaluated as $\mu_{r}=-1-j 0.23$, the WPT efficiencies are significantly decreased down to about $20 \%$ due to the power absorption by the slab. If a ring-type $R R$ is used, the effective imaginary part of the slab permeability can be designed to be smaller and the WPT efficiency can be enhanced to that degree.

This work was supported by Mid-career Researcher Program through the National Research Foundation of Korea (NRF) grant funded by the Korea government (No. 2012047938). 


\section{REFERENCES}

[1] A. Kurs, A. Karalis, R. Moffatt, J. D. Joannopoulos, P. Fisher, and M. Soljacic, "Wireless power transfer via strongly coupled magnetic resonances," Science, vol. 317, no. 5834, pp. 83-86, Jul. 2007.

[2] J. B. Pendry, A. J. Holden, D. J. Robbins, and W. J. Stewart, "Magnetism from conductors and enhanced nonlinear phenomena," IEEE Transactions on Microwave Theory and Techniques, vol. 47, no. 11, pp. 2075-2084, Nov. 1999.
[3] J. B. Pendry, "Negative refraction makes a perfect lens," Physical Review Letters, vol. 85, no. 18, pp. 3966-3969, Oct. 2000.

[4] M. N. O. Sadiku, Elements of Electromagnetics. New York, NY: Oxford University Press, 2001.

[5] D. Jeon and B. Lee, "Simplified modeling of ring resonators and split ring resonators using magnetization," Journal of Electromagnetics Engineering and Science, vol. 13, no. 2, pp. 134-136, Jun. 2013. 